\title{
Étude méthodologique d'un parcellaire d'exploitation agricole en tant que système
}

\author{
P Morlon 1, M Benoit 2 \\ 1 INRA, département de recherches sur les systèmes agraires et le développement, \\ 26, bd du docteur DPetitjean, 21000 Dijon; \\ 2 INRA, Station systèmes agraires et développement, Domaine du Joly, 88500 Mirecourt, France
}

(Reçu le 23 janvier 1990; accepté le 26 mai 1990)

\begin{abstract}
Résumé - Une nouvelle méthode est proposée pour évaluer les contraintes et atouts pour la production agricole d'un parcellaire d'exploitation, considéré comme un tout. Les productions et les contraintes sont d'abord hiérarchisées. Ces contraintes sont croisées dans un tableau, définissant des cases plus ou moins favorables pour chaque production. Toutes les parcelles de l'exploitation sont finalement rangées dans ce tableau. Ce tableau est utilisé pour mieux comprendre les relations entre le fonctionnement d'une exploitation et les contraintes ressenties par l'agriculteur sur son parcellaire; pour évaluer les conséquences de changements techniques (drainage, nouvel équipement ou matériel) ou territoriaux (acquisition de parcelles, échanges ou remembrement); il peut l'être pour comparer différentes exploitations d'une même région.
\end{abstract}

parcellaire / méthode graphique / utilisation du sol / système d'exploitation

Summary - Methodology for farm field pattern study. A new method is proposed to evaluate the constraints and assets for agricultural production, of a farm field pattern, considered as a whole. The production, and then the constraints, are organized into a hierarchy (table l); these constraints are crossed on a second chart, defining more or less favourable conditions for each production. Finally, all the fields of the farm are plotted on the chart. This chart is used to understand more fully the relation between the field pattern and the functioning of the farm system (1st example, table III and fig 1); to assess the effects of technical (eg drainage or new machinery) or territorial (eg plot acquisition or exchange) changes (2nd example, table $I V$ ); it can also be used to compare different farms in the same region.

field pattern / graphical method / land use / farming system

\section{INTRODUCTION}

Considérer le parcellaire d'une exploitation agricole comme un tout semble une évidence pour un agriculteur. Cependant, des opérations de recherche récentes ont confirmé l'intérêt de se donner des méthodes capables de caractériser cette totalité. Dans la lignée de travaux tels ceux menés à Rambervillers en 1972 (INRA-ENSSAA, 1973) où la description du territoire de chaque exploitation était intégrée à l'analyse de son fonctionnement, notre travail propose une formalisation opérationnelle des relations entre territoire et faits techniques (Gras et al, 1989).

Lors d'une enquête sur les transformations permises par le drainage dans des exploitations lorraines (Benoit et al, 1985), nous étions partis de l'idée, couramment admise à l'époque dans les milieux agricoles, que, lorsque les agriculteurs drainaient des prairies permanentes, c'était pour les remplacer par des cultures en rotation sur terres labourées. Mais l'enquête montra qu'après drainage, des parcelles proches de l'exploitation auparavant cultivées étaient très souvent maintenues, voire remises en prairies, pour en faire des parcs pâturés par les vaches laitières.

Avant drainage, l'excès d'eau était souvent incompatible avec cette utilisation, repoussée sur des parcelles plus saines mais plus éloignées. Inversement, sans être elles-mêmes drainées, des parcelles changeaient d'utilisation à la suite du drainage des autres. Ainsi, l'effet principal du drainage était une réorganisation de l'ensemble de l'utilisation du parcellaire de l'exploitation, plutôt qu'un simple passage des parcelles drainées de prairies en culture. 


\section{Comment prévoir et interpréter de telles évolutions ? Comment rendre compte de ces observations ? Quelles caracté- ristiques des parcelles et du parcellaire doivent être prises en considération, et de quelle façon?}

Des questions analogues se sont posées lors de suivis pluriannuels de la gestion du pâturage des vaches laitières : difficilement interprétables sans tenir compte des autres parcelles avec lesquelles ce pâturage interfère, ces suivis durent englober par nécessité toutes les parcelles en prairies, puis l'ensemble des opérations techniques sur toutes les parcelles de l'exploitation.

De ces démarches de recherche visant à évaluer l'impact d'un type de territoire sur le fonctionnement des exploitations est issu un tableau à double entrée permettant une représentation, puis un diagnostic des relations entre les caractéristiques des parcelles et leur utilisation au sein d'une exploitation agricole déterminée.

\section{MATÉRIEL ET MÉTHODE}

\section{Matériel}

La méthode proposée a été élaborée lors de travaux avec des exploitations agricoles familiales dans le Sud de la Lorraine.

Le milieu naturel est caractérisé par une alternance de plateaux calcaires, de cuestas et de fonds de vallées argileuses humides, entre 280 et $450 \mathrm{~m}$ d'altitude, et un climat océanique à tendance continentale, avec des précipitations de 700 à $1000 \mathrm{~mm}$ par an, ríparties en toutes saisons.

Les fermes disposent de quelques dizaines d'hectares de terres. Plus de la moitié des surfaces, en prairies permanentes, sert de pâturage estival à un troupeau bovin laitier, dont l'alimentation hivernale est à base d'ensilage de maïs ou d'herbe et de foin. La proportion de cultures de vente (blé, orge, colza) augmente avec la dimension de l'exploitation, l'élevage laitier ayant tendance à disparaître dans les plus grandes.

Intégré à la méthode de diagnostic d'exploitation agricole Digrex (Benoit et al, 1988), ce tableau a été utilisé dans d'autres régions d'élevage.

\section{Obtention des données}

II s'agit d'une enquête : c'est l'agriculteur qui fournit les informations; un crayon et du papier peuvent suffire.
La compréhension entre l'agriculteur et l'enquêteur, et par conséquent la richesse et la qualité des données, demandera en général que soit tracé, même de façon schématique, un plan parcellaire, sur fond de photographies aériennes ou, à défaut, de carte IGN au 1/25000 (éventuellement agrandie). Enfin, des bottes peuvent être utiles pour un tour de plaine.

\section{Principes méthodologiques}

Dans les 2 exemples (drainage et pâturage), les déterminants de ce que l'on observe sur une parcelle sont, en partie, situés ailleurs (sur d'autres parcelles, par exemple) : l'utilisation d'une parcelle, et les performances techniques qui y sont obtenues, ne dépendent pas seulement de ses caractéristiques propres (de ses potentialités définies par un observateur extérieur), mais aussi de ses relations (distance, complémentarité) avec les autres éléments du système.

Du point de vue de la méthode utilisée, cela a une conséquence essentielle : il est nécessaire de pouvoir caractériser toutes les parcelles de façon homogène, sur le même ensemble de variables. Ne pas respecter cette condition entraînerait des biais dans l'interprétation. C'est pourquoi, dans la méthode proposée ici, et pour la quasi-totalité des exploitations des régions où nous avons travaillé, nous n'utilisons pas les analyses de terre à ce niveau d'étude.

D'autre part, la méthode doit permettre des comparaisons entre exploitations. Le tableau qui en est l'outil ne doit donc pas, au départ être construit pour chaque exploitation individuellement. A l'opposé, il ne peut être universel. Le cadre du tableau est donc construit pour un ensemble d'exploitations similaires (type de système, OTEX*), rencontrant des contraintes du même ordre (sur une petite région agricole, par exemple, ou un groupe de petites régions similaires).

\section{CONSTRUCTION DU TABLEAU}

II s'agit de classer et hiérarchiser les contraintes liées au parcellaire, ressenties par les agriculteurs dans la conduite de leurs opérations. Cela suppose au préalable une hiérarchisation des priorités de ces agriculteurs :

- quelles sont les productions prioritaires?

- quelles sont, pour celles-ci, les opérations techniques les plus exigeantes quant aux conditions que doivent remplir les parcelles où elles se déroulent; enfin, quelles sont les techniques utilisées?

- quelles contraintes pèsent sur ces opérations (pouvant les interdire, les rendre difficiles, les retarder, en réduire l'efficacité) ? 
De façon très générale, ces contraintes peuvent se diviser en 2 groupes indépendants l'un de l'autre, ce qui permet de les croiser en un tableau à 2 entrées :

a) celles liées au terrain, pour des raisons :

- d'ordre physique : topographie (pente et exposition) et caractéristiques du sol (pierrosité, battance, excès d'eau, faible profondeur, etc);

- d'ordre juridique ou contractuel (même non formalisé), ou imposé par l'environnement de l'exploitation : interdiction de labourer de la part du propriétaire, zone de protection de captage d'eau, etc;

b) celles liées à la structure du territoire utilisé: dimension et forme des parcelles, distances entre elles et aux bâtiments, obstacles à franchir pour y accéder.

À l'intérieur de chacun de ces groupes, les caractéristiques susceptibles d'être rencontrées sur une parcelle sont ordonnées suivant un gradient général de contraintes croissantes, par partitions ou dichotomies successives (absence/présence de la contrainte), l'ordre des partitions correspondant à la hiérarchie des contraintes perçues par les agriculteurs dans la petite région.

Prenons l'exemple des systèmes de polyculture-élevage laitier en plaine dans le sud de la Lorraine; les opérations les plus exigeantes sont :
- le pâturage des vaches laitières;

- l'ensilage (maïs ou herbe);

- l'installation (du labour au semis) des cultures de vente et la moisson.

Les principales contraintes rencontrées sont (tableaux I et II) :

a) les contraintes liées au terrain (colonnes) :

- l'excès d'eau, quelles qu'en soient les causes et modalités (bas-fonds, mouillères de pente, etc). Sa présence est défavorable pour ces 3 opérations;

- la pente, pour les chantiers d'ensilage et de moisson;

- les sols séchants en été, réduisant la production (herbe pour le pâturage estival des vaches laitières, maïs ensilage, voire céréales de printemps);

b) les contraintes dues à la structure du territoire, distances et obstacles à traverser (lignes) :

- les vaches laitières font 2 fois par jour le trajet aller-retour entre les parcelles pâturées et la salle de traite. Suivant les observations faites dans la région étudiée (Pasquier, 1984), les éleveurs évitent de leur faire traverser une route à grande circulation ou un village où plusieurs troupeaux peuvent avoir à se croiser, ou de les faire marcher sur une trop grande distance : cela constitue un premier seuil séparant les parcelles

Tableau I. Identification des productions prioritaires, des opérations techniques les plus exigeantes, et des contraintes rencontrées. (Exemple des systèmes de polyculture-élevage laitier dans le Sud de la Lorraine).

\begin{tabular}{|c|c|c|}
\hline $\begin{array}{l}\text { FONCTIONS } \\
\text { PRODUCTIVES } \\
\text { PRIORITAIRAS }\end{array}$ & $\begin{array}{l}\text { OPERATIONS TECHNIQUES } \\
\text { EXIGEANTES }\end{array}$ & $\begin{array}{l}\text { CONTRAINTES ET FACTEURS } \\
\text { LIMITANTS }\end{array}$ \\
\hline \multirow[t]{2}{*}{$\begin{array}{l}\text { Lait } \\
\text { en été }\end{array}$} & Pâturage & $\begin{array}{c}\text { Excès d'eau } \\
\text { Sécheresse estivale } \\
\text { dimension, distance et accès parc. }\end{array}$ \\
\hline & Traite & $\begin{array}{l}\text { Distance et obstacles } \\
--->\text { Salle de traite }\end{array}$ \\
\hline $\begin{array}{l}\text { Lait } \\
\text { en hiver }\end{array}$ & Ensilage & $\begin{array}{c}\text { Excès d'eau } \\
\text { pente } \\
\text { Sécheresse estivale (maïs) } \\
\text { Distance et accès parcelles }\end{array}$ \\
\hline \multirow{2}{*}{$\begin{array}{l}\text { Cultures } \\
\text { d'hiver }\end{array}$} & Labour et semis & Excès d'eau \\
\hline & Récolte & $\begin{array}{c}\text { Pente } \\
\text { Dimension parcelles }\end{array}$ \\
\hline \multirow{2}{*}{$\begin{array}{l}\text { Cultures } \\
\text { de printemps }\end{array}$} & Labour et semis & Excès d'eau \\
\hline & Récolte & $\begin{array}{c}\text { Sécheresse estivale } \\
\text { Pente - dimension parcelles }\end{array}$ \\
\hline
\end{tabular}


Tableau II. Hiérarchisation des contraintes et définition des cases favorables aux opérations exigeantes (cas des systèmes de polyculture-élevage laitier, Sud de la Lorraine). (1) SE: Sécheresse estivale; (2) VL: vaches laitières; (3) P: petites; G: grandes.

\begin{tabular}{|c|c|c|c|c|c|c|c|c|c|c|}
\hline & \multicolumn{4}{|c|}{ SANS EXCES D'EAU } & \multicolumn{4}{|c|}{ AVEC EXCES D'EAU } \\
\hline & & & \multicolumn{2}{|c|}{ SANS PENTE } & \multicolumn{2}{|c|}{ AVEC PENTE } & \multicolumn{2}{|c|}{ SANS PENTE } & \multicolumn{2}{|c|}{ AVEC PENTE } \\
\hline & & & SANS S.E.(1) & AVEC S.E.(1) & SANS S.E.(1) & AVEC S.E.(1) & SANS S.E.(1) & AVEC S.E.(1) & SANS S.E.(1) & AVEC S.E. 1 \\
\hline \multirow{2}{*}{ 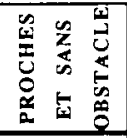 } & \multicolumn{2}{|c|}{$\mathbf{G}_{(3)}$} & \begin{tabular}{|l|} 
V.L (2), \\
Cultures, \\
ensilage
\end{tabular} & $\begin{array}{l}\text { V.L } \\
\text { (printemps), } \\
\text { Cultures d'hiver } \\
\text { ensilage }\end{array}$ & V.L & $\begin{array}{c}\text { V.L } \\
\text { (printemps) }\end{array}$ & & & & 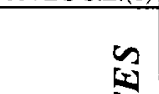 \\
\hline & \multicolumn{2}{|l|}{$\mathbf{P}$} & $\mathrm{V}, \mathrm{L}$ & V.L(printemps) & & & & & & $\geq$ \\
\hline \multirow{4}{*}{ 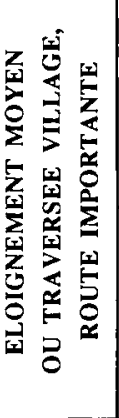 } & \multirow{4}{*}{\begin{tabular}{|l}
$\mathbf{G}$ \\
$\mathbf{R}$ \\
$\mathbf{A}$ \\
$\mathbf{N}$ \\
$\mathbf{D}$ \\
$\mathbf{E}$ \\
$\mathbf{S}$ \\
$\mathbf{P}$ \\
$\mathbf{E}$ \\
$\mathbf{T}$ \\
$\mathbf{I}$ \\
$\mathbf{T}$ \\
$\mathbf{E}$ \\
$\mathbf{S}$
\end{tabular}} & 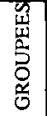 & $\begin{array}{l}\text { V.L (traite } \\
\text { au parc), } \\
\text { Cultures, } \\
\text { ensilage }\end{array}$ & $\begin{array}{l}\text { V.L (traite parc } \\
\text { printemps), } \\
\text { Cultures d'hiver } \\
\text { ensilage }\end{array}$ & $\begin{array}{l}\text { V.L Traite } \\
\text { au parc }\end{array}$ & $\begin{array}{l}\text { V.L Traite } \\
\text { au parc } \\
\text { printemps }\end{array}$ & & & & है \\
\hline & & 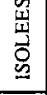 & $\begin{array}{l}\text { Cultures, } \\
\text { ensilage }\end{array}$ & $\begin{array}{l}\text { Cultures d'hiver } \\
\text { ensilage }\end{array}$ & & & & & & 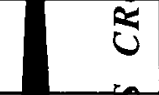 \\
\hline & & 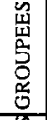 & & & & & & & & $\vdots$ \\
\hline & & 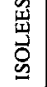 & & & & & & & & 5 \\
\hline \multirow{2}{*}{ 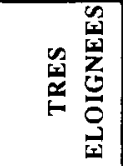 } & \multicolumn{2}{|l|}{ G } & Cultures & $\begin{array}{l}\text { Cultures } \\
\text { d'hiver }\end{array}$ & & & & & & \\
\hline & \multicolumn{2}{|l|}{$\mathbf{P}$} & & & CONTR & LINTES & CROISS & ANTES & & \\
\hline
\end{tabular}

favorables et défavorables au pâturage des vaches laitières.

- Un deuxième seuil, plus éloigné (Benoit, 1985), correspond à la distance (de l'étable) et aux obstacles au-delà desquels les agriculteurs évitent d'avoir à réaliser des chantiers d'ensilage.

Dans chaque exploitation, certaines parcelles sont jugées trop petites par l'exploitant pour certains chantiers, ou même pour y faire pâturer l'ensemble du troupeau de vaches laitières.

La construction du tableau définit des cases favorables ou plus ou moins défavorables aux opérations et donc aux productions sélectionnées au départ, et ceci au niveau auquel s'applique cette étape : grands types de système de production par petite région. Mais nous n'avons à aucun moment, jusqu'à présent, précisé les seuils séparant l'absence et la présence de chaque contrainte : ceux-ci sont en effet propres à chaque exploitation, leur comparaison entre exploitations (ou selon les techniques employées) étant une des principales utilisations possibles du tableau.

\section{REMPLISSAGE DU TABLEAU}

En s'aidant de préférence d'un plan ou schéma, il se fait, pour chaque exploitation, en dialoguant avec l'agriculteur : c'est lui qui indique si telle ou telle contrainte est limitante sur chaque parcelle, pourquoi et comment.

Concrètement, comment remplir le tableau ? Voici ce que nous faisons habituellement :

Dans chaque case sont notés l'identificateur (nom ou numéro), la surface et, grâce à des abréviations ou symboles (par exemple des couleurs), l'utilisation actuelle de chaque unité de terrain. Par unité de terrain, nous entendons des parcelles, morceaux ou groupes de parcelles qui présentent les mêmes contraintes par rapport à tous les critères et permettent par exemple des interventions ou exploitations faites dans la foulée aux mêmes dates.

Le remplissage est exhaustif et sans répétition: la somme des surfaces portées dans les cases est égale à la SAU totale de l'exploitation.

Des symboles indiquent également les contraintes dont la fréquence n'a pas été jugée suffisante pour créer des lignes ou colonnes (par exemple : absence de point d'eau, roches, ombrage d'une forêt ou exposition, etc). L'ensemble des données concernant une unité de terrain est mis entre crochets si l'une des contraintes entraîne un blocage complet : par exemple parcelle enclavée dans la zone bâtie du village ou inondée à chaque crue.

Dans une démarche de diagnostic global d'exploitation agricole (Benoit et al, 1988), ce rem- 
plissage permet une première évaluation des contraintes et atouts présentés par le parcellaire, pour les opérations techniques et donc les productions déterminées au départ.

Nous utilisons 2 règles choisies pour se libérer au maximum des hésitations qu'entraînerait la prise en compte des niveaux d'intensification et la fixation de seuils de chargement animal :

- si une des activités doit être réalisée en dehors des groupes de cases qui lui sont favorables, on décide qu'il y a contrainte : l'importance en est jugée à la fois sur la proportion de surface considérée et sur l'intensité et le nombre des contraintes;

- si des activités considérées dans la région comme moins exigeantes quant aux caractéristiques des parcelles (foins, pâturage de génisses ou de moutons, etc) occupent, durant toute la saison, des cases favorables à des activités considérées comme plus exigeantes (pâturage de vaches laitières, maîs ensilage, cultures de vente) on décide qu'il y a atout pour ces dernières, car elles disposent d'une réserve de surface pouvant leur être affectée (il faudra vérifier qu'il n'y a pas sur ces surfaces des contraintes cachées).

On peut s'apercevoir alors d'incohérences (apparentes), dont l'analyse, avec l'agriculteur, permet de mieux saisir ce qui guide ses pratiques d'utilisation des parcelles.

\section{RÉSULTATS ET UTILISATIONS}

\section{Deux exemples d'exploitations laitières en Lorraine}

\section{Quelle représentation l'agriculteur a-t-il de l'utilisation de son territoire?}

Le GAEC D (père-fils, 2 ménages) exploite 86 ha. La production principale, prioritaire, est le lait, qui fournit plus de $60 \%$ des rentrées monétaires, le reste provenant de la vente d'animaux d'élevage (veaux, génisses pleines, bœufs) et de céréales.

L'alimentation hivernale du troupeau des vaches laitières (effectif variant entre 40 et 45 ) est à base d'ensilage de maïs $(30 \mathrm{~kg}$ d'ensilage par vache et par jour), complété de foin et regain à volonté. L'été, le pâturage des prairies permanentes est complété par de l'ensilage d'herbe, du foin, et de la luzerne (affouragement en vert).
Les activités les plus exigeantes sont bien celles identifiées a priori au niveau régional :

- pâturage estival des laitières;

- ensilage d'herbe et maïs;

- installation et récolte des céréales, avec les pointes de travail : ensilage, fenaison, moisson.

Les parcelles, de 1 à 10 ha, sont dispersées (fig 1); les distances et obstacles à traverser les font regrouper de la façon suivante :

\section{0 ha dans un cercle de $3,5 \mathrm{~km}$ de diamètre}

$-13,5$ ha accessibles directement depuis la maison et les bâtiments anciens, situés dans le village;

- 29 ha de l'autre côté du village et de la route Vittel-Mirecourt-Nancy : les vaches y étaient traites au parc l'été, avant la construction d'une nouvelle étable de 60 places à $500 \mathrm{~m}$ de la sortie du village, à l'entrée d'un parc de 9 ha;

- 17 ha accessibles en empruntant ou traversant la route Vittel-Epinal.

\section{5 ha à $15 \mathrm{~km}$ en traversant Vittel et Contrexéville}

Lorsque l'on remplit le tableau II avec la hiérarchie des contraintes perçue au niveau de la petite région (excès d'eau en premier), on est frappé par le fait que les 7 ha d'ensilage d'herbe sont réalisés dans des conditions très différentes de celles définies a priori comme favorables : excès d'eau, éloignement et traversée de 2 agglomérations (tableau IIla).

La discussion à ce sujet avec l'agriculteur fait alors ressortir que c'est le manque d'herbe en été (parcelles séchantes), et non l'excès d'eau, qui est le plus fortement ressenti : par rapport aux terrains proches de l'exploitation, cette parcelle d'ensilage, située dans un bas-fond alluvial inondable, présente une croissance de l'herbe décalée et complémentaire : plus tardive d'un mois au printemps, mais se prolongeant tout l'été. Ce qui intéresse l'agriculteur est le regain abondant, et la pâture de fin d'été, qui permet de décharger les autres parcs en y mettant les vaches taries, les vêlages se faisant à l'automne. L'ensilage est la seule utilisation printanière permettant de profiter de ces avantages. II reste alors la question du chantier : en entraide avec les voisins, et en payant une entreprise, tout l'ensilage est réalisé en un jour. Si l'ensilage était réalisé sur des parcelles proches, il faudrait exploiter cette parcelle lointaine en foin, c'est-àdire y retourner plusieurs jours de suite, avec, 


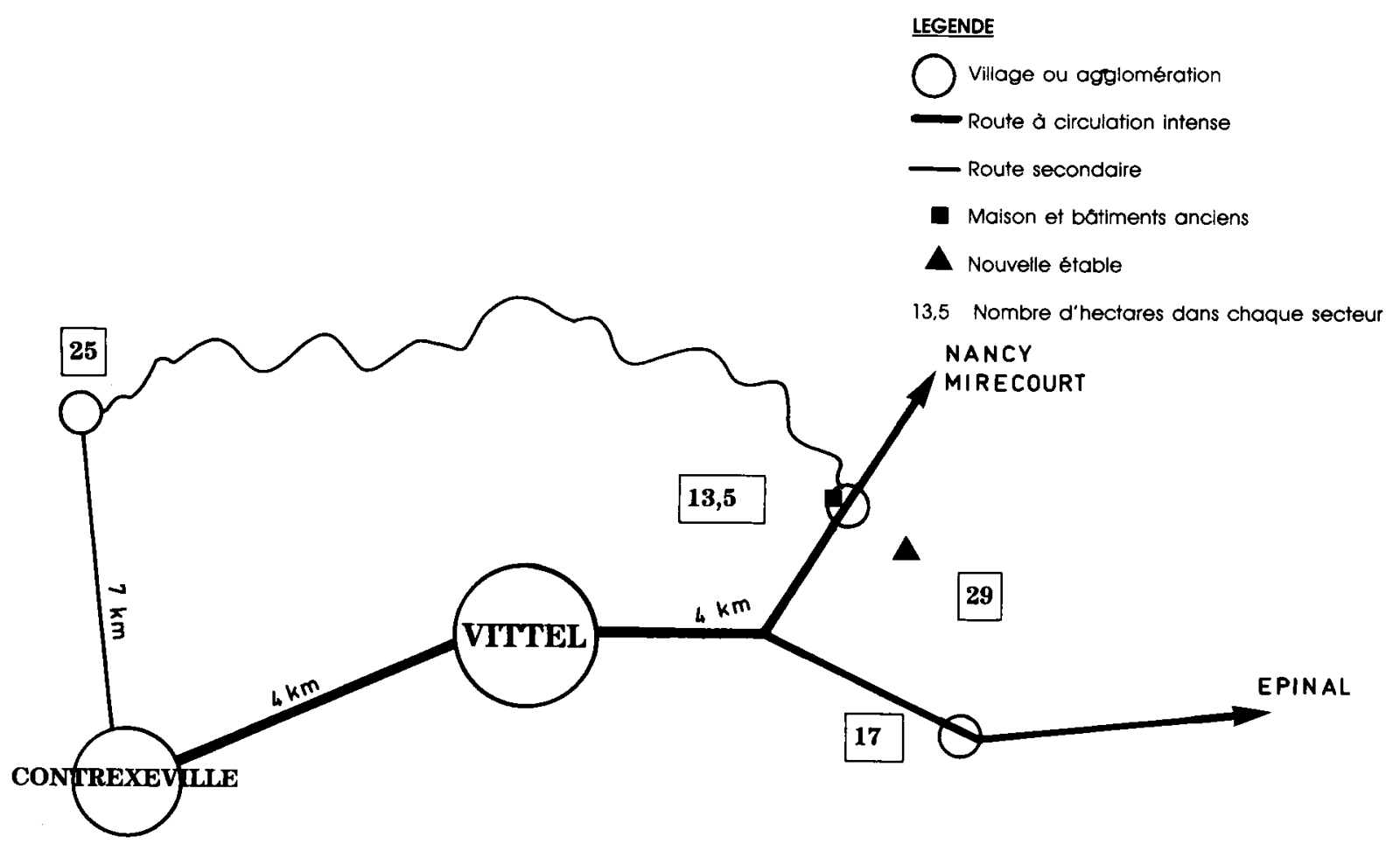

Fig 1. Schéma spatial de GAEC D.

de plus, le risque que le temps n'y soit pas le même (il peut faire beau ici, et pleuvoir là-bas). Le travail est rendu aux voisins à des périodes qui sont creuses pour le GAEC.

On reconstruit donc le tableau, par permutation des colonnes (intitulé et contenu), selon la hiérarchie des contraintes qui explique le mieux le fonctionnement de l'exploitation (tableau IIIb). Cette nouvelle version permet de mieux percevoir comment la contrainte éloignement est acceptée parce qu'elle permet de résoudre en partie la contrainte sécheresse estivale sur d'autres parcelles.

Ultérieurement, rien n'interdit de regarder de plus près pourquoi l'herbe ne pousse pas en été, et quelles techniques permettraient d'y remédier.

\section{Une stratégie d'acquisition, d'aménagement et d'utilisation des parcelles}

$M$ et Mme T, la quarantaine, 4 enfants, conduisent la seule exploitation intensive et en phase de croissance, dans une commune où plus de la moitié des agriculteurs sont âgés et sans succession.

À la fin du remembrement, en 1973, les parcelles dispersées sur une moitié du territoire communal (du même côté que la maison et l'étable), sont très facilement accessibles : moins de $2 \mathrm{~km}$, sans traversée de village ni route importante. A cette date, les 69 ha se répartissent en 20,5 de prairies incultivables, 26,5 de prairies cultivables et 22 de terres labourées.

Parmi les parcelles qui se libèrent dans la commune, lesquelles choisir pour s'agrandir ? Celles dont le sol est le plus favorable (sain, profond, sans pierres...) ? Le tableau IV montre que ce n'est pas ce critère qui a guidé les choix. Les parcelles nouvelles sont choisies non pas en raison de leurs qualités intrinsèques (parfois très médiocres : excès d'eau dans le bas, taillis sur pierriers sur le plateau...), mais pour relier entre elles les parcelles antérieures afin de constituer trois groupes, dont le troisième représentait, dès 1985,50 ha d'un seul tenant (seulement coupé par un chemin de remembrement) :

- le premier, très proche de l'étable et sur sols profonds au pied du coteau, pour le pâturage des vaches laitières;

- le deuxième, sur le plateau calcaire, a des sols sains et parfois un peu séchants, qui seraient favorables à une céréaliculture de vente si le climat (à $450 \mathrm{~m}$ d'altitude) n'y était un peu froid; il est donc cultivé pour l'alimentation du troupeau : une moitié en rotation céréales-maïs ensilage, et l'autre en mélange luzerne-dactyle (ensilage et 2 regains); 
Tableau Illa. GAEC D. Répartition des parcelles, avec l'excès d'eau comme première contrainte. Les rectangles représentent les surfaces des parcelles, et leur contiguité (dans une même case du tableau), à l'exclusion de toute autre caractéristique. P: pâturage ( $L$ vaches laitières, $T$ vaches taries, $G$ génisses, $B$ mâles boucherie); $E$ : ensilage; F: foin; R: regain; V: affouragement en vert ( $\mathrm{Z}$ luzerne, $\mathrm{H}$ herbe); $\mathrm{C}$ : cultures en rotation ( $M$ incluant maïs ensilage). Une flèche mince indique un changement d'utilisation ou de caractéristiques.

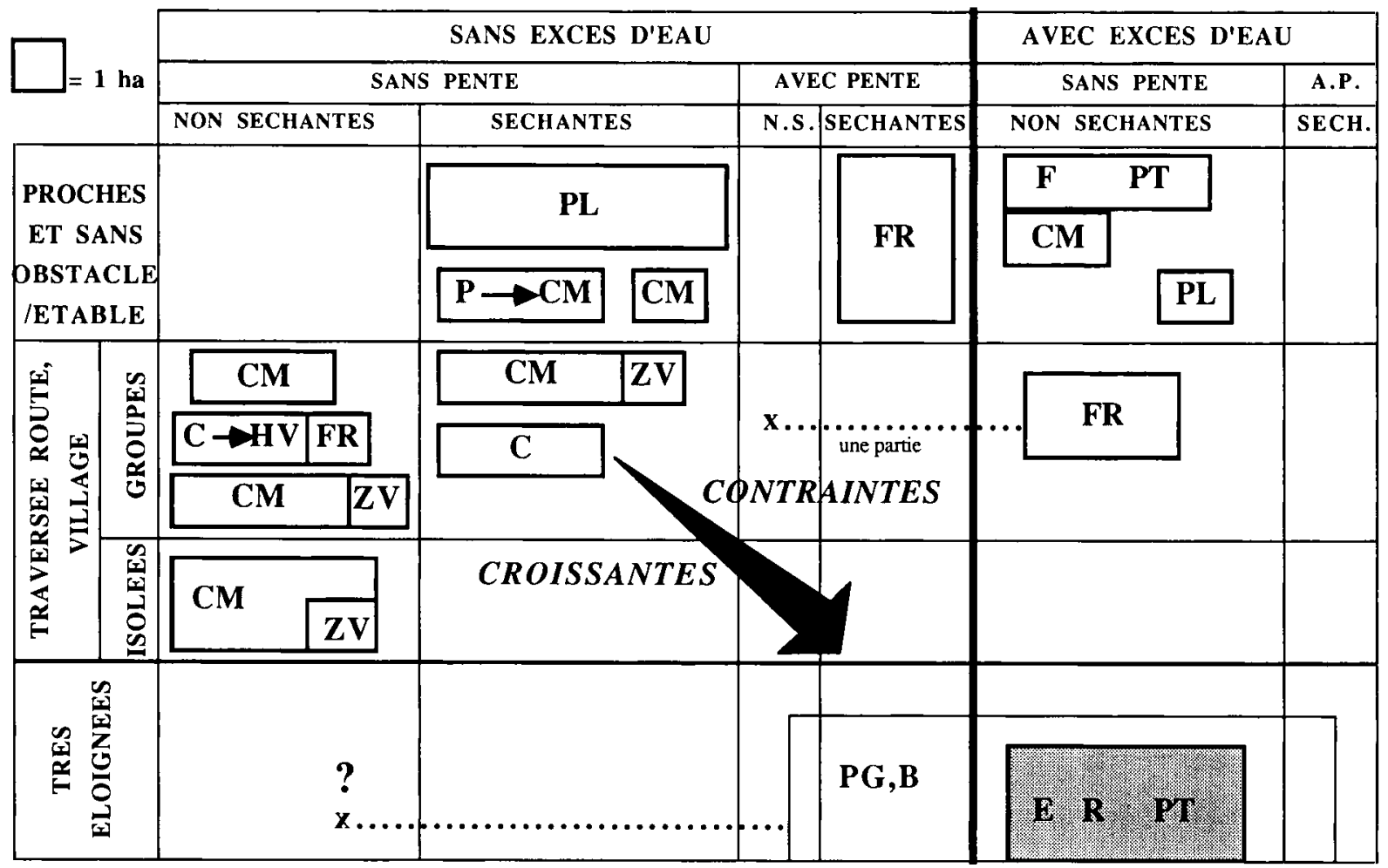

Tableau IIIb. GAEC D. Répartition des parcelles avec la sécheresse estivale comme $1^{\text {re }}$ contrainte. Même légende que tableau Illa.

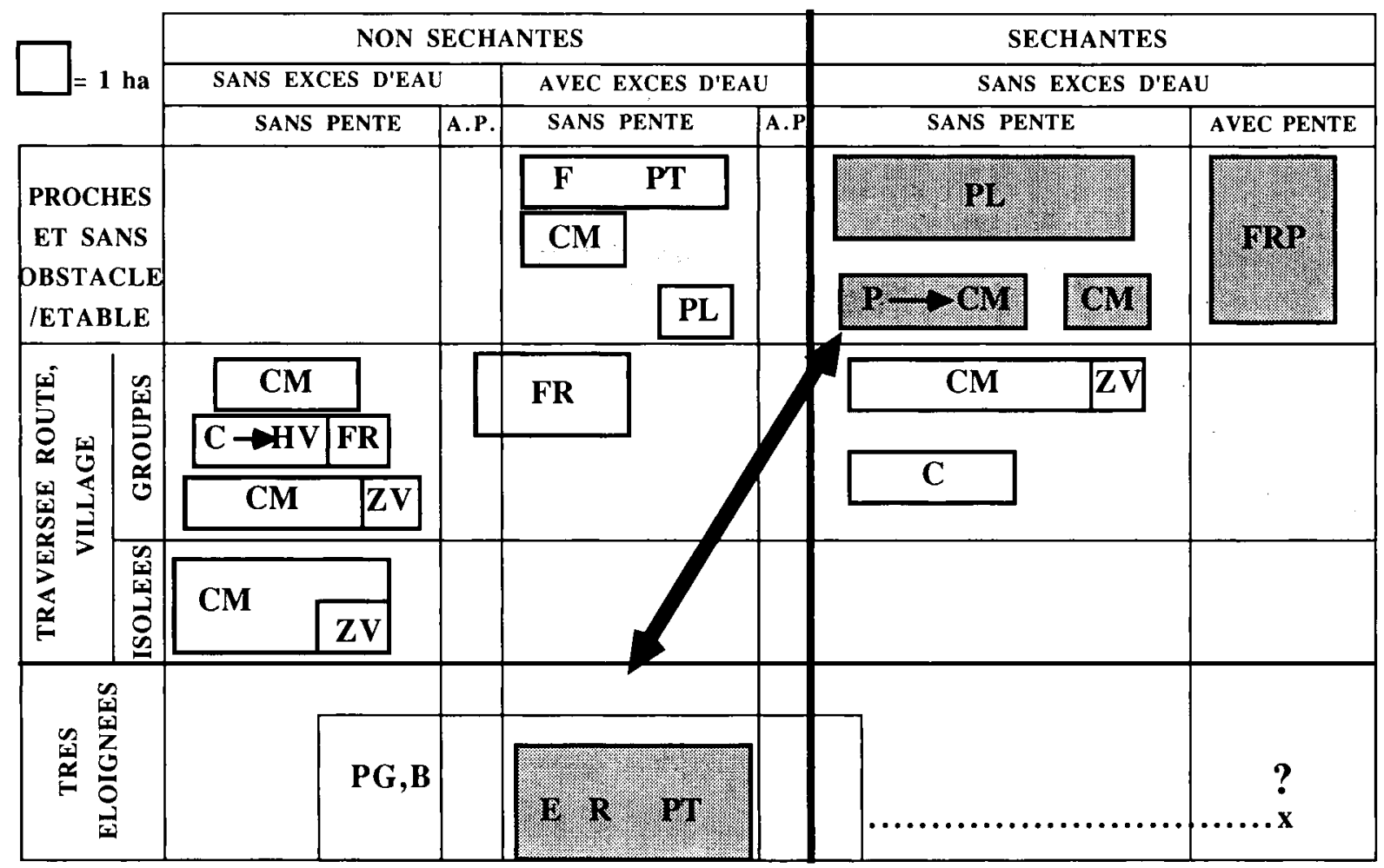


Tableau IV. Stratégie foncière exploitation T: drainages et acquisitions / locations de parcelles. Même légende que tableau IIla. Les dates en caractères gras entourées d'un cercle sont celles d'acquisition des nouvelles parcelles.

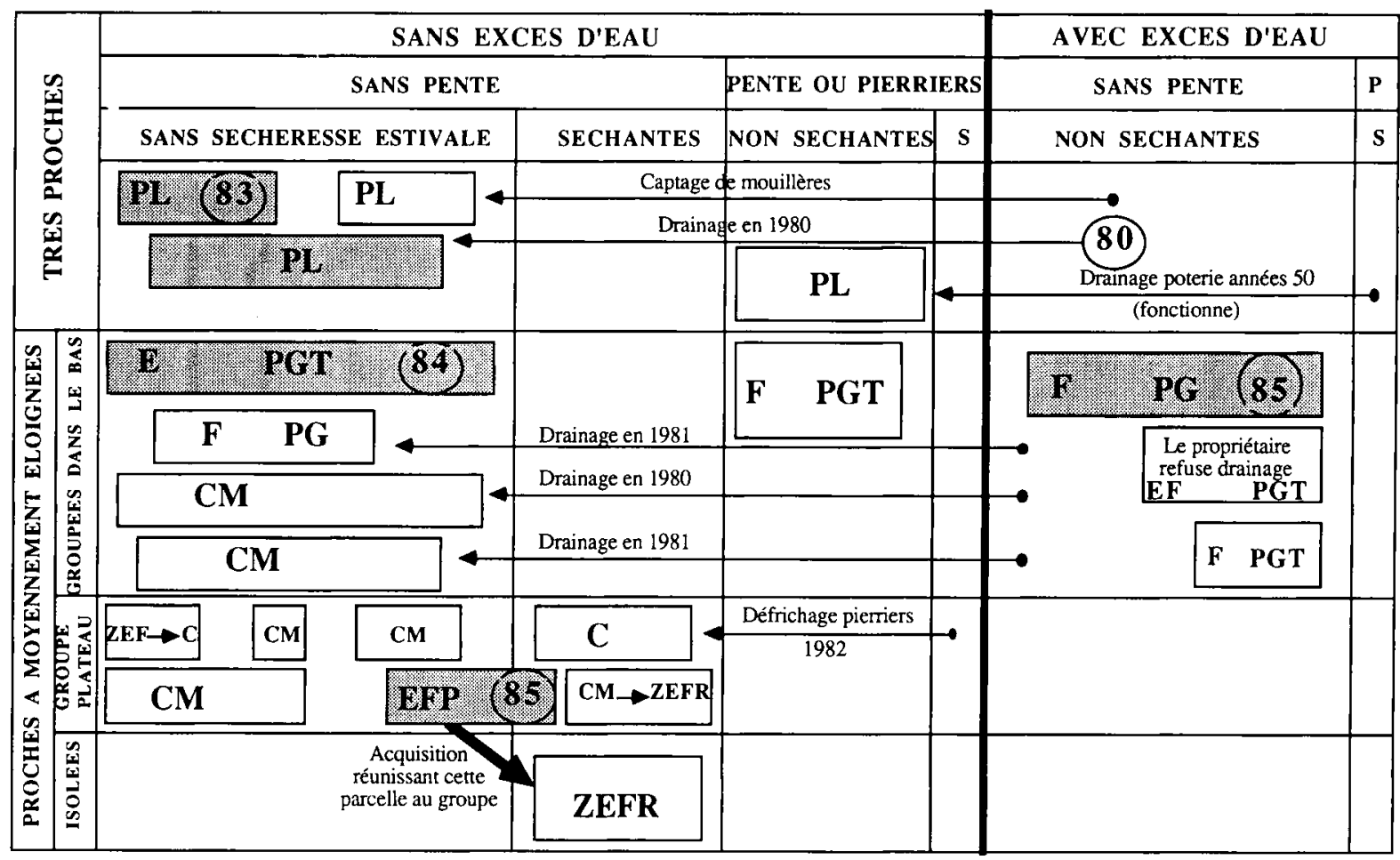

- le troisième, le plus grand, est situé au pied du coteau, comme le premier mais plus loin. L'augmentation régulière du troupeau de vaches laitières (28 vaches en 1974, 55 en 1982, 70 en 1985) devait aboutir à ce que le premier groupe devienne trop petit pour les alimenter, obligeant à les faire pâturer sur ce troisième groupe (avec traite au parc à cause de la distance) : c'est dans cette perspective que 12,5 ha de prairies $y$ ont été drainés en 1980-1981.

Mais les quotas laitiers, s'ils n'ont pas au départ remis en cause l'augmentation du troupeau, ont conduit à écarter cette solution rigide et à en adopter une autre, beaucoup plus souple et adaptable à d'éventuelles modifications du système :

- maintenir les laitières sur le premier groupe exclusivement, avec un complément d'alimentation estivale : foin, ensilage d'herbe ou de luzerne, affouragement en vert (luzerne-dactyle et, en fin d'été, détourage du maïs);

- utiliser le troisième groupe pour le pâturage des génisses, des vaches taries, et d'éventuels animaux à viande, et en labourer une partie pour une rotation maïs ensilage-céréales, après drainage.

Le défrichement des pierriers sur le plateau, et dans le bas le drainage (réalisé quel que soit le mode de faire-valoir de la parcelle, à moins d'une opposition du propriétaire), réduisent les contraintes pour les utilisations actuelles, et rendent possibles, pour l'avenir, des changements d'utilisation, selon l'évolution du système.

\section{DISCUSSION}

\section{Place dans la démarche agronomique}

Les caractéristiques retenues pour décrire les parcellaires d'exploitations agricoles sont celles qui interfèrent avec la mise en œuvre des pratiques agricoles actuelles. Ces caractéristiques renvoient aux concepts de potentialités agricoles ou potentialités techniques (Auricoste et al, 1983). Actuellement, elles concernent essentiellement la faisabilité de techniques agricoles et l'élaboration du rendement de productions agricoles; leurs homologues peuvent être cherchés dans d'autres directions : conditions de travail, sensibilité à la pollution. Notre proposition est donc, en l'état actuel, à référer aux schémas d'élaboration des rendements et aux potentialités agricoles (BTI, 1985).

Le contenu de chaque case, correspondant à des caractéristiques de parcelles données, est une liste de cultures explicitées par de grands 
traits de leurs conduites. Nous sommes dans le cadre d'une explicitation simple des systèmes de cultures (Sébillotte, 1988) : les successions de cultures et les techniques appliquées à chacune d'elles. Ainsi le tableau représente l'ensemble des systèmes de culture qui coexistent dans une exploitation et leurs positions respectives dans ce cadre de contraintes.

\section{Place dans la démarche \\ de recherche sur le fonctionnement des exploitations agricoles}

«Si l'hypothèse d'une invariance de la structure paramétrée (donc de l'organisation) est longtemps commode, elle ne l'est pas toujours. Elle devient même un handicap inacceptable dès lors que nous voulons rendre compte de l'aptitude d'un système à s'adapter à de nouvelles stabilités par rapport à de nouvelles finalités. Si nous voulons rendre compte de cette aptitude dans nos systémographies des objets sociaux et des objets socio-techniques, il nous faut considérer que les structures aussi varient : autrement dit que le système reçoit et restitue aux processus, dans lesquels il intervient, des variables structurelles" (Le Moigne, 1984).

C'est dans ce cadre que notre contribution méthodologique se situe : expliciter la structure parcellaire pour que cette information soit traitée par le système de décision. Celui-ci ensuite la maintiendra ou la modifiera en fonction de ses finalités. Les déplacements des parcelles et des seuils, évoqués ci-dessous, en sont une illustration.

Nous avons choisi volontairement un premier exemple où le tableau est reconistruit spécialement pour une exploitation donnée à un moment donné. Cela permet une compréhension de la complexité du fonctionnement de l'exploitation, mais pas la comparaison entre plusieurs exploitations (le calage de la diversité), ou entre étapes séparées par des changements techniques importants dans la vie d'une exploitation. Or, même s'il peut être utilisé à d'autres fins, ce tableau a été conçu pour permettre des comparaisons.

C'est pour cela que son cadre n'est pas construit pour une exploitation déterminée, mais au niveau d'un type de système dans une petite région. Si ce cadre représente bien la hiérarchie des priorités et des contraintes ressenties par l'agriculteur, il est logique que celui-ci cherche au cours du temps à améliorer l'adéquation entre les caractéristiques de son parcellaire et les exi- gences de ses productions prioritaires. Cela se traduit, dans le tableau, par des déplacements :

- déplacements "verticaux" des parcelles, c'est-à-dire modifications de la structure spatiale de l'exploitation par remembrement, échanges à l'amiable, achats ou ventes, prise ou abandon de locations, etc; ou par construction d'une nouvelle étable près des parcs à vaches laitières;

- déplacements "horizontaux" des parcelles (de la droite vers la gauche, avec la convention que nous avons adoptée), c'est-à-dire suppression ou réduction d'une contrainte, par drainage, irrigation, épierrement, sous-solage, amendements; qu'il s'agisse d'échanger (ou d'acquérir) des parcelles, ou d'en aménager, pourquoi celleci plutôt que celle-là ? Le tableau est un outil de raisonnement et de dialogue : dans quelles cases, favorables à quelle(s) activité(s), cela permet-il d'augmenter ou de regrouper les surfaces?

- déplacements des seuils : la règle énoncée au départ - pour chaque parcelle, c'est l'agriculteur qui indique si telle ou telle contrainte est limitante, pourquoi et comment, fixant ainsi les seuils de distance, pente, etc - est conforme à la démarche systémique. Mais on s'aperçoit bien entendu que ces seuils diffèrent (plus ou moins) d'une exploitation à l'autre, ou se modifient au cours du temps sur la même exploitation.

Les exemples les plus simples, assez triviaux, sont liés à des choix de techniques :

- pente : tracteurs de montagne, autochargeuses, etc;

- portance : roues cages ou jumelées, pneus larges, etc;

- déplacements des vaches laitières : traite au parc (c'est la fréquence de cette technique dans la région qui justifie, dans le tableau, la troisième partition : isolées ou groupées, pour les parcelles moyennement éloignées).

Sur le tableau, le changement d'une technique employée se traduit non pas, comme précédemment, par le déplacement d'une ou plusieurs parcelles d'une case à une autre mais, si l'on peut dire, par le déplacement de la séparation entre lignes ou colonnes défavorables (ou contraintes) et favorables, ces dernières englobant plus de parcelles avec une technique qu'avec une autre.

Travailler à technique identique réduit la variabilité des seuils, sans la supprimer. Les niveaux d'exigence, ou de risques acceptés, diffèrent en effet entre agriculteurs, entre exploitations. Un cas particulier en est la relativisation d'une 
contrainte par une autre plus forte, comme dans le premier exemple où une contrainte forte sur les parcelles proches, a considérablement repoussé la distance jusqu'à laquelle l'agriculteur accepte d'ensiler. En ce sens, l'acquisition de nouvelles parcelles, qui modifie au moins les distances (en créant par exemple de nouveaux groupes de parcelles) peut aussi modifier les seuils perçus par l'agriculteur, soit par comparajson des caractéristiques des anciennes et des nouvelles parcelles, soit en modifiant la hiérarchie des contraintes.

Ces seuils dépendent, enfin, de l'ensemble de la gestion, de la maîtrise technique de chaque agriculteur : ainsi, la comparaison des tableaux remplis pour des exploitations apparemment similaires, mais dont les résultats sont très différents, peut être un outil de recherche pour trouver les causes de ces différences.

\section{CONCLUSION}

Cette proposition de systémographie des parcellaires d'exploitations agricoles s'inscrit dans une volonté de modéliser les relations qui unissent les activités agricoles pilotées par un acteur dans un territoire (Lardon et al, 1990). Ces modèles sont centraux pour trois points de vue :

- celui de laménageur : comment choisir les portions de territoire à aménager et pourquoi choisir tel aménagement ?

- celui du développeur : dans quelle mesure le territoire est-il un frein à l'évolution des exploitations?

- celui qui élabore des outils destinés à évaluer le territoire d'une exploitation à des fins de reprise. En effet, le nombre de reprises autres que père-fils augmente et la pression intrafamiliale à l'évaluation correcte d'une exploitation lors de partages successoraux reste importante. À moyen terme, l'éventualité d'un marché des exploitations à reprendre et l'entrée en scène plus importante d'acheteurs-agriculteurs. de la CEE est à envisager avec des demandes induites sur le jugement de la qualité d'un ensemble parcellaire, corps de ferme et bâtiments.

Une étape ultérieure visera à fournir à partir de cette démarche un outil régional de caractérisation des territoires d'exploitations agricoles à travers une typologie des parcellaires et un mo- dèle régional liant chaque type de parcellaire à une gamme de système de culture (Sébillotte, 1988) d'une part, et à une gamme de types de fonctionnement d'exploitations agricoles, d'autre part (Cristofini, 1986).

\section{RÉFÉRENCES}

Auricoste C, Deffontaines JP, Fiorelli JL, Langlet A, Osty PL (1983) Friches, parcours et activités d'élevage. Point de vue d'agronomes sur les potentialités agricoles. Le cas des Vosges et des Causses. Versailles, INRA Publications, $55 \mathrm{p}$

Benoit M (1985) La gestion territoriale des activités agricoles. L'exploitation et le village : deux échelles d'analyse en région d'élevage. Thèse de DocteurIngénieur INA-PG, $152 p$

Benoit M, Morlon P, Teilhard de Chardin B (1985) Transformations permises par le drainage dans les exploitations lorraines. In: Séminaire de la Commission d'Agrométéorologie de I'INRA. Octobre 1985. Conditions et effets des excès d'eau, 459-469

Benoit $M$, Brossier J, Chia E, Marshall E, Morlon $P$, Roux M, Teilhard de Chardin B (1988) Diagnostic global d'exploitation agricole : une proposition méthodologique. Études et recherches sur les systèmes agraires et le développement 12, $47 \mathrm{p}$

BTI (1985) Eléments pour la connaissance et la mise en valeur de la montagne, 399-401

Cristofini (1986) La petite région vue à travers le tissu de ses exploitations : un outil pour l'aménagement et le développement rural. Études et recherches sur les systèmes agraires et le développement 6, $43 p$

Gras R, Benoit $M$, Deffontaines JP, Duru M, Lafarge M, Langlet A, Osty PL (1989) Le fait technique en agronomie. Activité agricole, concepts et méthodes d'étude. INRA-L'Harmattan, $184 \mathrm{p}$

Lardon S, Deffontaines JP, Baudry J, Benoit M (1990) L'espace est aussi ailleurs. Études et recherches sur les systèmes agraires et le développement (sous presse)

INRA-ENSSAA (1972) Conditions du choix des techniques de production et évolution des exploitations agricoles, Région de Rambervillers, SEI, INRA, $160 p$

Le Moigne JL (1984) La théorie du système général. Théorie de la modélisation. PUF, $320 \mathrm{p}$

Pasquier L (1984) La traite au pâturage. Importance et insertion dans les exploitations laitières lorraines. Mémoire ENSA Rennes. INRA-SAD VersaillesDijon-Mirecourt, $110 \mathrm{p}$

Sébillotte $M$ (1988) Les systèmes de culture. Encyclopediae Universalis 5, 884-887 